\title{
Prognostic Value of the Regional Lymph Node Station in Pancreatoduodenectomy for Ampullary Carcinoma
}

\author{
KOSEI TAKAGI, YASUO NAGAI, YUZO UMEDA, RYUICHI YOSHIDA, KAZUHIRO YOSHIDA, \\ TOMOKAZU FUJI, KENJIRO KUMANO, KAZUYA YASUI, TAKAHITO YAGI and TOSHIYOSHI FUJIWARA \\ Department of Gastroenterological Surgery, Okayama University Graduate School of Medicine, \\ Dentistry, and Pharmaceutical Sciences, Okayama, Japan
}

\begin{abstract}
Background/Aim: The optimal extent of lymph node dissection for ampullary carcinoma is controversial. The aim of this study was to investigate the efficacy of lymph node dissection for ampullary carcinoma. Patients and Methods: Between 2000 and 2020, a total of 75 patients undergoing radical resection for ampullary carcinoma were included. The efficacy index (EI) was calculated by multiplication of the frequency of lymph node metastasis (LNM) at the station and the 5-year survival rate of patients with metastasis at the station. Results: Out of 75 patients, 14 had LNM. The EI for the peri-pancreatic head (station 13 and 17) and superior mesenteric artery (station 14) lymph node were 4.4 and 3.5, respectively. Whereas the peri-gastric (station 5 and 6), common hepatic artery (station 8), and liver hilum (station 12) lymph node stations had zero EI. Although the number of patients with the station 16 dissected was small (9\%), the para-aortic (station 16) lymph nodes had the highest EI of 14.3 despite being distant lymph nodes. Conclusion: We identified the distribution of LNM and survival benefit of lymph node dissection for ampullary carcinoma. Our results suggest that the optimal extent of lymph node dissection for ampullary carcinoma could be reconsidered.
\end{abstract}

Ampullary carcinoma of the duodenum, or carcinoma of the papilla of Vater, is a rare malignant neoplasm arising from the region of Vater's ampulla. Radical resection with

This article is freely accessible online.

Correspondence to: Kosei Takagi, MD, Ph.D., Department of Hepato-Biliary and Pancreatic Surgery, Okayama University Hospital, 2-5-1 Shikata-cho, Kita-ku, Okayama 700-8558, Japan. Tel. +81 862237151, Fax: +81 862218775, e-mail: kotakagi15@gmail.com

Key Words: Ampullary carcinoma, carcinomas of the papilla of Vater, lymph node dissection, outcome. pancreatoduodenectomy is the standard treatment for ampullary carcinoma (1); however, the significance of the optimal lymph node dissection on outcomes remains unclear. Although the Japanese guideline for ampullary carcinoma has proposed the optimal extent of lymph node dissection (2), the impact of the lymph node station dissected on the prognostic survival benefit is still unknown (3). Furthermore, data on long-term outcomes such as survival and recurrence patterns are limited $(4,5)$.

The efficacy index (EI) has been proposed as a useful index to evaluate the therapeutic value of lymph node dissection for gastric cancer (6), and validated for various gastrointestinal and hepatopancreatobiliary cancers (7-9). However, to date, the impact of lymph node dissection on outcomes using the EI has not yet been examined in patients following radical resection for ampullary carcinoma.

The aim of this study was to evaluate the efficacy of lymph node dissection using the EI as well as to determine the optimal extent of lymph node dissection for ampullary carcinoma. Moreover, long-term outcomes including survival and recurrence patterns were investigated.

\section{Patients and Methods}

Patients and study design. The study protocol was approved by the Ethics Committee at our institution (approval no. 2110-003) and conducted according to the Declaration of Helsinki. We performed a retrospective review of the clinical database including 75 patients who underwent radical resection with pancreatoduodenectomy for ampullary carcinoma at the Okayama University Hospital (Okayama, Japan) between January 2000 and December 2020.

Clinical data. Using the database, the following clinicopathological data were extracted: age, sex, body mass index, the American Society of Anesthesiologists (ASA) physical status, operative time, blood loss, postoperative complications, pathological factors, recurrence (presence or absence), recurrence pattern, and outcome at the last follow-up (survival or death). Pathological factors were assessed by pathologists in accordance with the General Rules for Clinical and Pathological Studies on Cancer of the Biliary Tract of Japan (2). 
The lymph node stations investigated in this study were categorized as the following regions: peri-gastric lymph node (station 5 and 6), common hepatic artery (CHA) lymph node (station 8), liver hilum lymph node (station 12), peri-pancreatic head lymph node (station 13 and 17), superior mesenteric artery (SMA) lymph node (station 14), and para-aortic lymph node (station 16). The regional lymph node stations included the peri-gastric, CHA, liver hilum, peri-pancreatic head, and SMA lymph nodes. In contrast, the para-aortic lymph node was considered as the distant lymph node station.

Regarding postoperative recurrence, the following recurrence patterns were investigated: local recurrence, liver metastasis, lung metastasis, peritoneal dissemination, and distant lymph node metastasis (LNM). Local recurrences included LNM around the pancreatic head, and recurrences in the remnant pancreas and around the reconstructions. Recurrences of LNM at the para-aortic, Virchow, and mediastinal lymph node were defined as distant LNM. Moreover, the swimmer plot was utilized to visualize post-recurrent clinical courses for patients with recurrences.

Efficacy of lymph node dissection for each lymph node station. Frequency of LNM was defined as the number of patients with LNM at the station divided by the number of patients in whom the station was dissected. The dissection of each lymph node station included both en bloc and sampling retrieval of the area. The efficacy of lymph node dissection for each station was investigated using the EI, by multiplication of the frequency of LNM at the station and the 5-year survival rate of patients with LNM for each station as an index of survival benefit (6).

Perioperative management and follow-up protocol. Our standard protocol included no neo-adjuvant and adjuvant chemotherapy for ampullary carcinoma. In patients with advanced TNM stage, adjuvant chemotherapy can be performed based on the decision of the multidisciplinary team. All patients received follow-up examination every 3 to 6 months at the outpatient clinic with respect to their physical condition and recurrence.

Statistical analysis. Data are presented as mean with standard deviation for continuous variables, and proportions for categorical data. Overall (OS), recurrence-free (RFS), and post-recurrence (PRS) survival rates were calculated using the Kaplan-Meier method. RFS is defined as the time from surgery to recurrence or death. The log rank test was used to analyze differences between curves. JMP version 11 software (SAS Institute, Cary, NC, USA) was used for statistical analysis.

\section{Results}

Study cohort. The clinicopathological characteristics of the 75 patients included are demonstrated in Table I. Our cohort included 43 male and 32 female patients with a mean age of 70.3 years. The mean operative time and estimated blood loss for pancreatoduodenectomy were 421 minutes and 430 $\mathrm{ml}$. Postoperative outcomes included the incidences of mortality and major complications of $0 \%$ and $13.3 \%$, respectively. Regarding pathological factors, $\mathrm{T}$ factor was as follows: Tis $(n=8)$; T1 $(n=22)$; T2 $(n=25)$; and T3 $(n=20)$. Following a mean number of 14.5 lymph nodes dissections,
Table I. Characteristics of patients undergoing pancreatoduodenectomy for ampullary carcinoma between 2000 and 2020.

\begin{tabular}{|c|c|}
\hline Variables & \\
\hline No. of patients & 75 \\
\hline \multicolumn{2}{|l|}{ Preoperative factor } \\
\hline Age (year) & $70.3(9.0)$ \\
\hline Gender (Male/Female) & $43(57 \%) / 32(43 \%)$ \\
\hline BMI $\left(\mathrm{kg} / \mathrm{m}^{2}\right)$ & $22.1(3.4)$ \\
\hline ASA-PS $(1 / 2 / 3)$ & $9(12 \%) / 54(72 \%) / 12(16 \%)$ \\
\hline \multicolumn{2}{|l|}{ Intraoperative factor } \\
\hline Operative time (min) & $421(89)$ \\
\hline Blood loss (ml) & $430(467)$ \\
\hline \multicolumn{2}{|l|}{ Postoperative factor } \\
\hline Mortality & $0(0 \%)$ \\
\hline Major complication & $10(13 \%)$ \\
\hline \multicolumn{2}{|l|}{ Pathological factor } \\
\hline $\mathrm{T}$ stage $(\mathrm{Tis} / 1 / 2 / 3)$ & $\begin{array}{l}8(11 \%) / 22(29 \%) / \\
25(33 \%) / 20(27 \%)\end{array}$ \\
\hline Dissected LN & $14.5(10)$ \\
\hline Lymph node metastasis $(+/-)$ & $14(19 \%) / 61(81 \%)$ \\
\hline \multicolumn{2}{|l|}{ TNM stage } \\
\hline Stage 0 (TisNOM0) & $8(11 \%)$ \\
\hline Stage IA (T1aNOM0) & $9(12 \%)$ \\
\hline Stage IB (T1b/T2N0M0) & $31(41 \%)$ \\
\hline Stage IIA (T3aNOM0) & $7(9 \%)$ \\
\hline Stage IIB (T3bNOM0) & $6(8 \%)$ \\
\hline Stage IIIA (T1/T2/T3N1M0) & $10(13 \%)$ \\
\hline Stage IIIB (TanyN2M0) & $2(3 \%)$ \\
\hline Stage IV (TanyNanyM1) & $2(3 \%)$ \\
\hline \multicolumn{2}{|l|}{ Survival } \\
\hline Follow-up period (year) & $5.3(4.4)$ \\
\hline OS $(1-/ 3-/ 5-/ 10-y)$ & $95.9 \% / 76.5 \% / 70.5 \% / 61.4 \%$ \\
\hline RFS (1-/3-/5-/10-y) & $87.6 \% / 65.5 \% / 61.5 \% / 53.1 \%$ \\
\hline
\end{tabular}

BMI: Body mass index; ASA-PS: American Society of Anesthesiologists physical status; LN: lymph node; OS: overall survival; RFS: recurrence-free survival. Data are presented as means ( \pm standard deviation) or numbers (percentages).

LNM was detected in 14 patients out of 75 . Furthermore, microvascular invasion was identified in 34 patients.

Following a mean follow-up of 5.0 years, the 1-, 5-, and 10 -year survival rates were $97.2 \%, 70.3 \%$, and $60.6 \%$ for OS, and $87.5 \%, 61.2 \%$, and $52.2 \%$ for RFS (Figure 1). OS based on TNM stage is also demonstrated in Figure 1B. The 5 -year OS was $100 \%$ for stage $0,83 \%$ for stage I, $65 \%$ for stage II, and $57 \%$ for stage III and IV.

Incidence of lymph node metastasis and survival benefit at each node station. Details on the frequency of LNM, the 5year OS rate of patients with LNM at the station, and the EI are demonstrated in Table II. Out of dissected lymph nodes areas, LNM was found frequently in the peri-pancreatic head lymph nodes (13.3\%). However, there were no LNM in the peri-gastric and CHA lymph nodes. The para-aortic lymph node was dissected in seven patients, and two patients had 
A

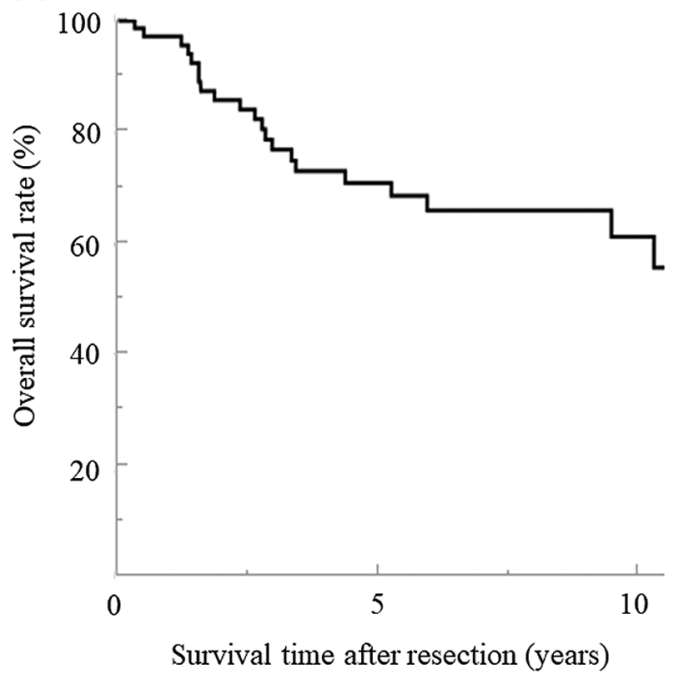

B

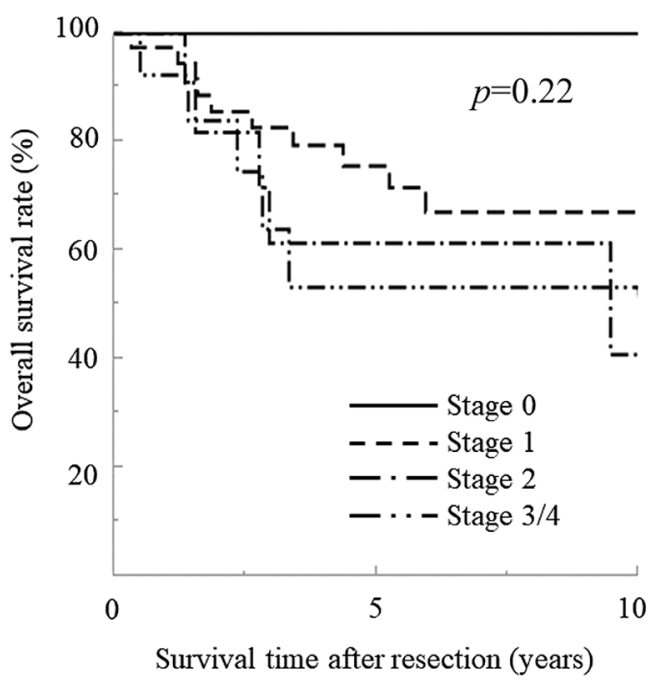

Figure 1. Overall survival curve following radical resection (A), stratified by TNM stage (B).

Table II. Index of estimated survival benefit from lymph node dissection.

\begin{tabular}{|c|c|c|c|c|c|}
\hline Station & $\begin{array}{l}\text { No. of patients } \\
\text { with LN dissection }\end{array}$ & $\begin{array}{c}\text { No. of patients } \\
\text { with LNM }\end{array}$ & $\begin{array}{l}\text { Incidence } \\
\text { of LNM* }\end{array}$ & 5-year OS & $\begin{array}{c}\text { 5-year } \\
\text { OS index }(E I)^{\dagger}\end{array}$ \\
\hline Peri-gastric LN (\#5/6) & $27(36 \%)$ & 0 & $0 \%$ & N.A. & $0^{\ddagger}$ \\
\hline CHA LN (\#8) & $60(80 \%)$ & 0 & $0 \%$ & N.A. & $0 \neq$ \\
\hline Liver hilum LN (\#12) & $61(81 \%)$ & 1 & $1.6 \%$ & N.A. & $0 \ddagger$ \\
\hline Peri-pancreatic head LN (\#13/17) & $75(100 \%)$ & 10 & $13.3 \%$ & $32.8 \%$ & $4.4 \ddagger$ \\
\hline SMA LN (\#14) & $37(49 \%)$ & 4 & $10.5 \%$ & $33.3 \%$ & $3.5 \div$ \\
\hline Para-aortic LN (\#16) & $7(9 \%)$ & 2 & $28.6 \%$ & $50 \%$ & 14.3 \\
\hline
\end{tabular}

LN: Lymph node; LNM: lymph node metastasis; OS: overall survival; CHA: common hepatic artery; SMA: superior mesenteric artery. *The number of patients with LNM at the station/the number of patients undergoing LN dissection. †The incidence of LNM to the station $\mathrm{x}$ the 5-year survival

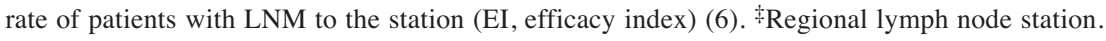

LNM at the para-aortic lymph nodes, diagnosed as having distant metastasis with stage IV.

The 5-year OS rate with LNM in the peri-pancreatic head, the SMA, and the para-aortic lymph node stations were $32.8 \%, 33.3 \%$, and $50 \%$, respectively. With regards to the EI assessment, the peri-pancreatic head and the SMA lymph node stations had the survival benefit of lymph node dissection among the regional lymph node stations, whereas the peri-gastric, CHA, and liver hilum lymph node stations had zero EI. Although the number of patients with the station 16 dissected was small, the para-aortic lymph nodes had the highest EI of 14.3 despite being distant lymph nodes.

Recurrence pattern following radical resection. Out of 75 patients, $21(28.0 \%)$ had recurrences following radical
Table III. Recurrence patterns following radical resection for ampullary carcinoma.

\begin{tabular}{lc}
\hline Variables \\
\hline No. of patients \\
Recurrence (+/-) \\
Initial recurrence site & 75 \\
$\quad$ Local recurrence & \\
Liver metastasis & 6 \\
Lung metastasis & 6 \\
Peritoneal dissemination & 1 \\
Distant lymph node metastasis & 1 \\
Multiple-site & 5 \\
Survival & 2 \\
PRS (1-/2-/3-y) & \\
\hline
\end{tabular}

PRS: Post-recurrence survival. 


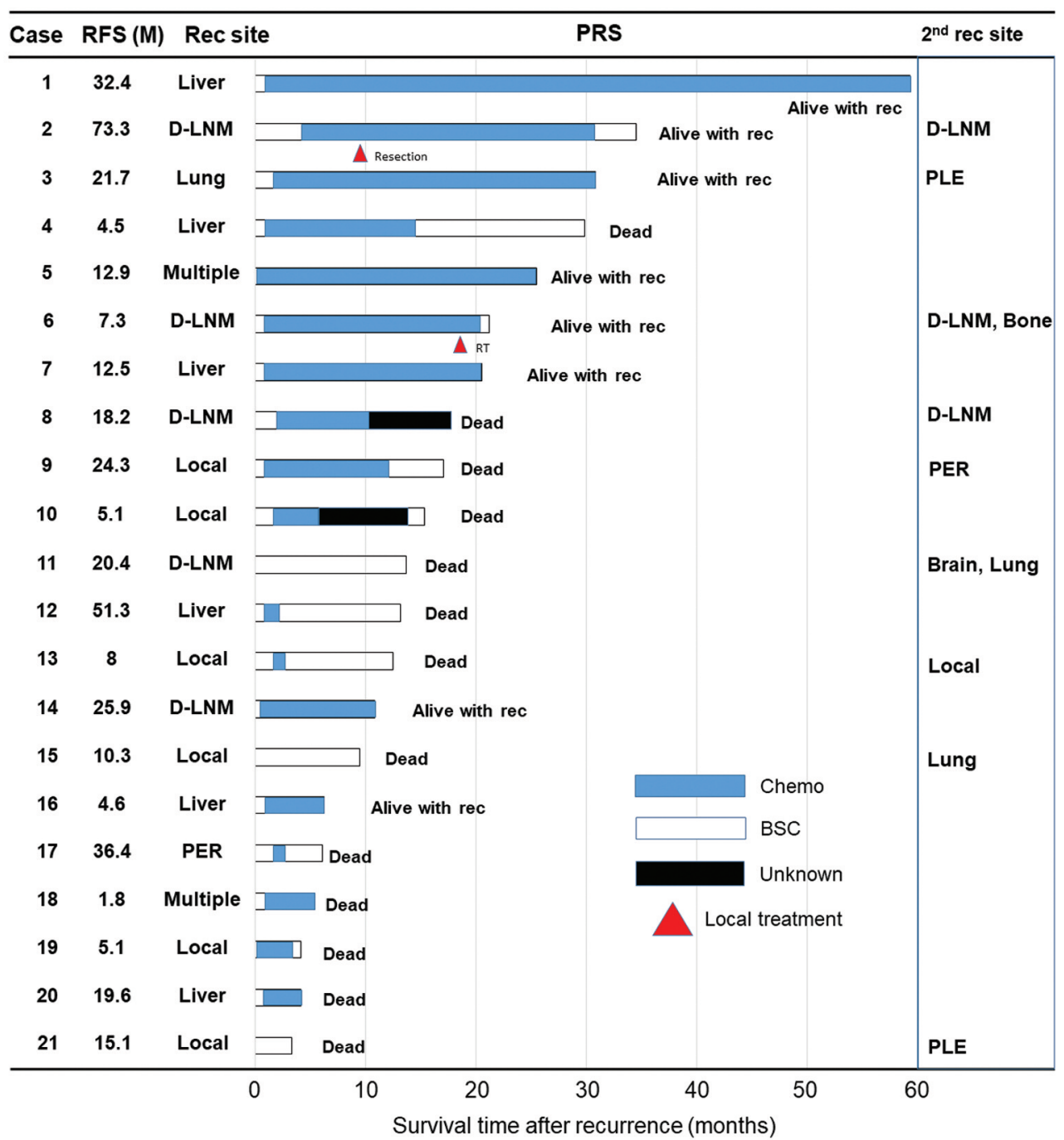

Figure 2. Swimmer plot demonstrating post-recurrence clinical courses. RFS: Recurrence-free survival; PRS: post-recurrence survival; D-LNM: distant lymph node metastasis; PLE: pleural dissemination; PER: peritoneal dissemination; BSC: best supportive care.

resection (Table III). Among 21 patients with recurrences, the initial recurrence sites included local recurrence $(n=6)$, liver metastasis $(n=6)$, lung metastasis $(n=1)$, peritoneal dissemination $(n=1)$, distant LNM $(n=5)$, and multiple-site $(n=2)$. The 1-, 2-, and 3-year PRS were $66.6 \%, 27.7 \%$, and $20.8 \%$, respectively.

Detailed post-recurrent clinical courses for patients with recurrence are demonstrated in the swimmer plot (Figure 2). The mean interval from surgery to recurrence was 19.6 months. Regarding post-recurrent treatment, most of patients underwent chemotherapy for recurrence sites, whereas three patients (14\%) received the best supportive care only. The median PRS was 13.6 months. The 1-year PRS was significantly better in patients with chemotherapy than those with the best supportive care $(65.6 \%$ vs. $0 \%, p<0.001)$, as shown in Figure 3.

\section{Discussion}

The present study is the first to investigate the efficacy of lymph node dissection using the EI in patients undergoing radical resection for ampullary carcinoma. Although little has been examined regarding the distribution of LNM and the optimal extent of lymph node dissection for ampullary carcinoma, the results suggested that several regions of the regional lymph nodes had no prognostic benefit on lymph node dissection, whereas lymph node dissection of a distant lymph node station (station 16) might have a survival benefit. Furthermore, longterm survival and recurrence patterns following radical resection were investigated in patients with ampullary carcinoma.

According to the EI assessment, lymph node dissection of the peri-gastric, $\mathrm{CHA}$, and liver hilum lymph node stations 


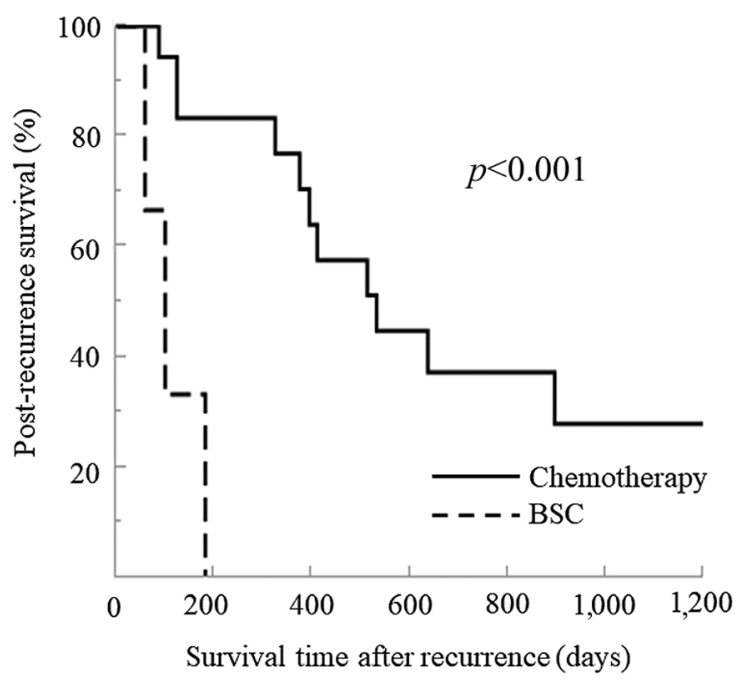

Figure 3. Post-recurrence survival curves in patients receiving chemotherapy versus best supportive care. BSC: Best supportive care.

might be waived based on the findings showing low incidence of LNM as well as zero EI in these stations. In contrast, the survival benefit of lymph node dissection was found in the peri-pancreatic head and the SMA lymph node stations. A recent study suggested that the peri-pancreatic head lymph nodes (station 13 and 17) and other should not be categorized equally among the regional lymph nodes (3); however, we revealed the impact of each lymph station on outcomes in more detail. Moreover, our results suggested that LNM at the para-aortic lymph node station should not be contraindicated for radical resection of ampullary carcinoma. In fact, the para-aortic lymph node station had higher EI than the regional lymph node stations. However, the SMA lymph node dissection was not performed in half of the patients, and the para-aortic lymph node dissection was performed only in $9 \%$ of patients. Therefore, further investigation should be conducted to clarify the significance of lymph node dissection in the stations 14 and 16 .

With respect to long-term outcomes following radical resection for ampullary carcinoma, OS and RFS in this study were relatively better than those of previous reports $(1,10$, 11). In addition, better PRS was confirmed in the present study (12). Looking at recurrence patterns as shown in Table III, approximately $70 \%$ of patients with recurrence had distant metastases including liver, lung, peritoneal or lymph node metastases. Although the evidence of adjuvant chemotherapy after radical resection has been open to debate, a recent meta-analysis has suggested the safety and efficacy of adjuvant therapy in high-risk patients or those with the pancreaticobiliary subtype of ampullary carcinoma (13). Therefore, adjuvant chemotherapy for high-risk patients could be associated with increased RFS and OS. With regard to systemic therapy for recurrence, our results suggested that systematic chemotherapy should be considered based on the findings that patients with the best supportive care had poorer outcomes. However, the choice of chemotherapy regimen is still controversial due to lack of evidence. In this study, various chemotherapy regimens were used, such as the combination of gemcitabine with cisplatin, S1, or cisplatin and S1 (14). Accordingly, further investigation with high evidence-based trials should be performed in order to provide more evidence regarding biological and histological features, and therapeutic strategies (15).

Several limitations should be acknowledged in this study. As ampullary carcinoma is a rare cancer, the sample size is relatively small. In addition, this study is retrospective, and a single-center study. Therefore, our findings might not be applied to those at other centers. To obtain further evidence on the efficacy of lymph node dissection as well as longterm outcomes following radical resection, a multi-center study with larger sample size should be performed.

In conclusion, this study identified the distribution of LNM and survival benefit of lymph node dissection for ampullary carcinoma. The peri-pancreatic head (station 13 and 17) and the SMA (station 14) lymph node dissection was associated with survival benefit, whereas no survival benefit was found in the peri-gastric (station 5 and 6), CHA (station 8 ), and liver hilum (station 12) lymph node stations. Our results suggested that the optimal extent of lymph node dissection for ampullary carcinoma could be reconsidered; however further investigation is required. Last, but certainly not least, relatively good long-term outcomes following radical resection were confirmed, even after recurrence.

\section{Conflicts of Interest}

The Authors declare no conflicts of interest regarding this study.

\section{Authors' Contributions}

K.T. contributed to the study conception and design, acquisition of data, development of the protocol, and drafting of the manuscript. Y.N., K.Y. T.F. K.K. and K.Y contributed to the acquisition of data and development of the protocol. Y.U. and R.Y. contributed to the analysis and interpretation of the quantitative data, and the drafting of the manuscript. T.Y and T.F. contributed to the development of the protocol and the critical revision of the final draft. All Authors have approved the final version.

\section{References}

1 Winter JM, Cameron JL, Olino K, Herman JM, de Jong MC, Hruban RH, Wolfgang CL, Eckhauser F, Edil BH, Choti MA, Schulick RD and Pawlik TM: Clinicopathologic analysis of ampullary neoplasms in 450 patients: implications for surgical strategy and long-term prognosis. J Gastrointest Surg 14(2): 379387, 2010. PMID: 19911239. DOI: 10.1007/s11605-009-1080-7 
2 Japanese Society of Hepato-Biliary-Pancreatic Surgery: General Rules for Clinical and Pathological Studies on Cancer of the Biliary Tract (7th edition). Japan, Kanehiora Publ Corp., 2021.

3 Tashiro K, Kuroki N, Einama T, Iwasaki T, Miyata Y, Aosasa S, Inoue Y, Takahashi Y, Ogata S, Ueno H, Hase K, Yamamoto J and Kishi Y: Prognostic significance of regional lymph node metastasis according to station in ampullary carcinoma. J Hepatobiliary Pancreat Sci 27(10): 712-720, 2020. PMID: 32578342. DOI: 10.1002/jhbp.791

4 Balachandran P, Sikora SS, Kapoor S, Krishnani N, Kumar A, Saxena R and Kapoor VK: Long-term survival and recurrence patterns in ampullary cancer. Pancreas 32(4): 390-395, 2006 PMID: 16670621. DOI: 10.1097/01.mpa.0000220864.80034.63

5 Woo SM, Ryu JK, Lee SH, Yoo JW, Park JK, Kim YT, Jang JY, Kim SW, Kang GH and Yoon YB: Recurrence and prognostic factors of ampullary carcinoma after radical resection: comparison with distal extrahepatic cholangiocarcinoma. Ann Surg Oncol 14(11): 3195-3201, 2007. PMID: 17710498. DOI: 10.1245/s10434-007-9537-y

6 Sasako M, McCulloch P, Kinoshita T and Maruyama K: New method to evaluate the therapeutic value of lymph node dissection for gastric cancer. Br J Surg 82(3): 346-351, 1995 PMID: 7796005. DOI: 10.1002/bjs.1800820321

7 Katai H, Ishikawa T, Akazawa K, Fukagawa T, Isobe Y, Miyashiro I, Oda I, Tsujitani S, Ono H, Tanabe S, Nunobe S, Suzuki S, Kakeji Y and Registration Committee of the Japanese Gastric Cancer Association: Optimal extent of lymph node dissection for remnant advanced gastric carcinoma after distal gastrectomy: a retrospective analysis of more than 3000 patients from the nationwide registry of the Japanese Gastric Cancer Association. Gastric Cancer 23(6): 1091-1101, 2020. PMID: 32419120. DOI: 10.1007/s10120-020-01081-5

8 Imamura T, Yamamoto Y, Sugiura T, Okamura Y, Ito T, Ashida $\mathrm{R}$, Ohgi K and Uesaka K: Reconsidering the optimal regional lymph node station according to tumor location for pancreatic cancer. Ann Surg Oncol 28(3): 1602-1611, 2021. PMID: 32862372. DOI: $10.1245 / \mathrm{s} 10434-020-09066-5$

9 Cai MZ, Lv CB, Cai LS and Chen QX: Priority of lymph node dissection for advanced esophagogastric junction adenocarcinoma with the tumor center located below the esophagogastric junction. Medicine (Baltimore) 98(51): e18451, 2019. PMID: 31861019. DOI: 10.1097/MD.0000000000018451
10 Vilhordo DW, Gregório C, Valentini DF Jr, Edelweiss MIA, Uchoa DM and Osvaldt AB: Prognostic factors of long-term survival following radical resection for ampullary carcinoma. J Gastrointest Cancer 52(3): 872-881, 2021. PMID: 32808236. DOI: $10.1007 / \mathrm{s} 12029-020-00479-9$

11 Lian PL, Chang Y, Xu XC, Zhao Z, Wang XQ and Xu KS: Pancreaticoduodenectomy for duodenal papilla carcinoma: A single-centre 9-year retrospective study of 112 patients with long-term follow-up. World J Gastroenterol 23(30): 5579-5588, 2017. PMID: 28852317. DOI: 10.3748/wjg.v23.i30.5579

12 Bowitz Lothe IM, Kleive D, Pomianowska E, Cvancarova M, Kure E, Dueland S, Gladhaug IP and Labori KJ: Clinical relevance of pancreatobiliary and intestinal subtypes of ampullary and duodenal adenocarcinoma: Pattern of recurrence, chemotherapy, and survival after pancreatoduodenectomy. Pancreatology 19(2): 316-324, 2019. PMID: 30713128. DOI: 10.1016/j.pan.2019.01.019

13 Vo NP, Nguyen HS, Loh EW and Tam KW: Efficacy and safety of adjuvant therapy after curative surgery for ampullary carcinoma: A systematic review and meta-analysis. Surgery 170(4): 12051214, 2021. PMID: 33902926. DOI: 10.1016/j.surg.2021.03.046

14 Nagino M, Hirano S, Yoshitomi H, Aoki T, Uesaka K, Unno M, Ebata T, Konishi M, Sano K, Shimada K, Shimizu H, Higuchi R, Wakai T, Isayama H, Okusaka T, Tsuyuguchi T, Hirooka Y, Furuse J, Maguchi H, Suzuki K, Yamazaki H, Kijima H, Yanagisawa A, Yoshida M, Yokoyama Y, Mizuno T and Endo I: Clinical practice guidelines for the management of biliary tract cancers 2019: The 3rd English edition. J Hepatobiliary Pancreat Sci 28(1): 26-54, 2021. PMID: 33259690. DOI: 10.1002/jhbp. 870

15 Rizzo A, Dadduzio V, Lombardi L, Ricci AD and GadaletaCaldarola G: Ampullary carcinoma: an overview of a rare entity and discussion of current and future therapeutic challenges. Curr Oncol 28(5): 3393-3402, 2021. PMID: 34590592. DOI: 10.3390/ curroncol28050293 